\title{
A THERMAL ANALYSIS MODEL FOR HIGH POWER DENSITY BEAM STOPS*
}

\author{
S. Virostek, D. Oshatz, J. Staples, \\ LBNL, Berkeley, CA 94720, USA
}

\begin{abstract}
The Lawrence Berkeley National Laboratory (LBNL) is presently designing and building the $2.5 \mathrm{MeV}$ injector for the Spallation Neutron Source (SNS). The design includes various beam intercepting devices such as beam stops and slits. The target power densities can be as high as $500 \mathrm{~kW} / \mathrm{cm}^{2}$ with a beam stopping range of 25 to 30 microns, producing stresses well above yield in most materials. In order to analyze the induced temperatures and stresses, a finite element model has been developed. The model has been written parametrically to allow the beam characteristics, target material, dimensions, angle of incidence and mesh densities to be easily adjusted. The heat load is applied to the model through the use of a 3dimensional table containing the calculated volumetric heat rates. The load is based on a bi-gaussian beam shape which is absorbed by the target according to a Bragg peak distribution. The results of several analyses using the SNS Front End beam are presented.
\end{abstract}

\section{INTRODUCTION}

The SNS is an accelerator-based user facility which will produce pulsed beams of neutrons for use in scattering experiments. The project, a collaboration of six US national laboratories, is funded by the US Department of Energy and is currently under construction at Oak Ridge National Laboratory (ORNL). LBNL has designed and is fabricating the SNS Front End [1], which will accelerate a $38 \mathrm{~mA}, 6 \%$ duty factor $\mathrm{H}^{-}$beam to $2.5 \mathrm{MeV}$ for injection into a $1 \mathrm{GeV}$ linac. The Front End consists of an ion source and low energy beam transport line (LEBT) [2], a radio frequency quadrupole (RFQ) [3,4] and a medium energy beam transport line (MEBT) [5].

The Front End design contains several different beam intercepting devices including an emittance scanner slit, a beam chopping target (Figure 1) and a beam stop. The peak power density of the beam is up to $500 \mathrm{~kW} / \mathrm{cm}^{2}$ during the $1 \mathrm{~ms}$ long pulse. A beam of this intensity impinging on a target at a normal incidence will produce stresses well above the yield stress in virtually all materials. The power density incident on the target can be significantly decreased by reducing the angle of beam interception from normal incidence to just a few degrees.
This, in combination with the selection of a material having the optimal combination of thermal and mechanical properties, allows the design of a target which can survive the short term peak temperatures and stresses as well as long term fatigue effects.

This paper will summarize the details of a finite element model developed to predict the temperatures and stresses in various beam intercepting devices. The results of studies using numerous target materials, beam intensities and angles of incidence will be presented. Also, a comparison of results obtained using a Bragg peak heat load distribution versus that obtained with all heat applied at the surface will be given.

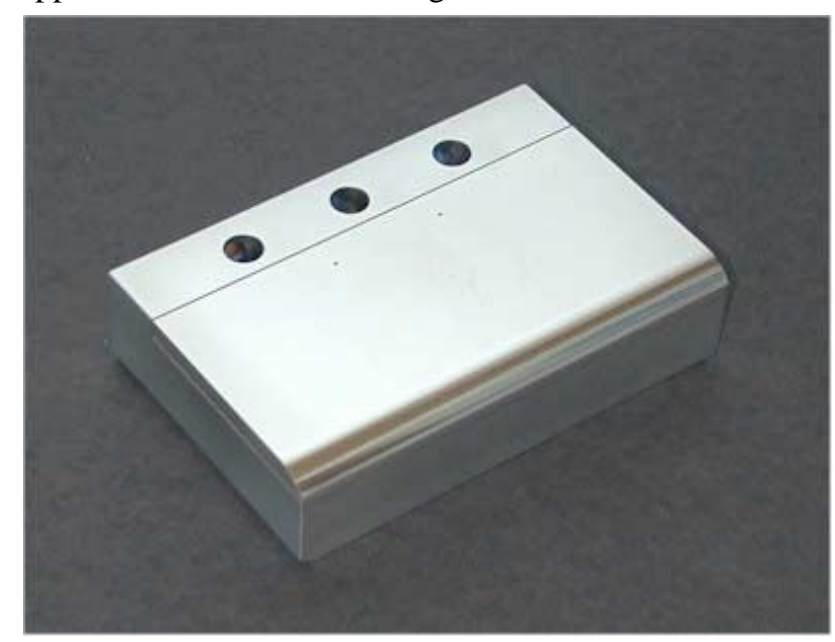

Figure 1: Beam chopper target made of TZM moly alloy.

\section{MODEL DESCRIPTION}

\subsection{Overview}

In order to better understand the performance limits of beam intercepting devices, an ANSYS $^{\odot}$ finite element model has been developed to predict the transient temperature and stress distributions within the target material. The model simulates the target with 3D solid thermal elements configured as a flat plate with quarter symmetry and uniform cooling on the back side. Upon obtaining the thermal solution, the elements are converted to structural elements for stress analysis. A key feature of the model is the method of application of the heat load. A $3 \mathrm{D}$ table is generated based on the bi-gaussian distribution

\footnotetext{
* This work is supported by the Director, Office of Science, Office of Basic Energy Sciences, of the U.S. Department of Energy under Contract No. DE-AC03-76SF00098.
} 
of the beam and the deposition of heat into the target for a given material and beam energy. The volumetric heat load table is generated and applied completely independent of the model mesh. An algorithm within ANSYS automatically superimposes the 3D table values on the model and applies heat to individual nodes as appropriate. This technique allows the load to be applied to any model geometry without the necessity of tracking either the numbering or positions of the nodes. Figure 2 shows a typical ANSYS temperature contour plot from an analysis of the SNS beam chopper target.

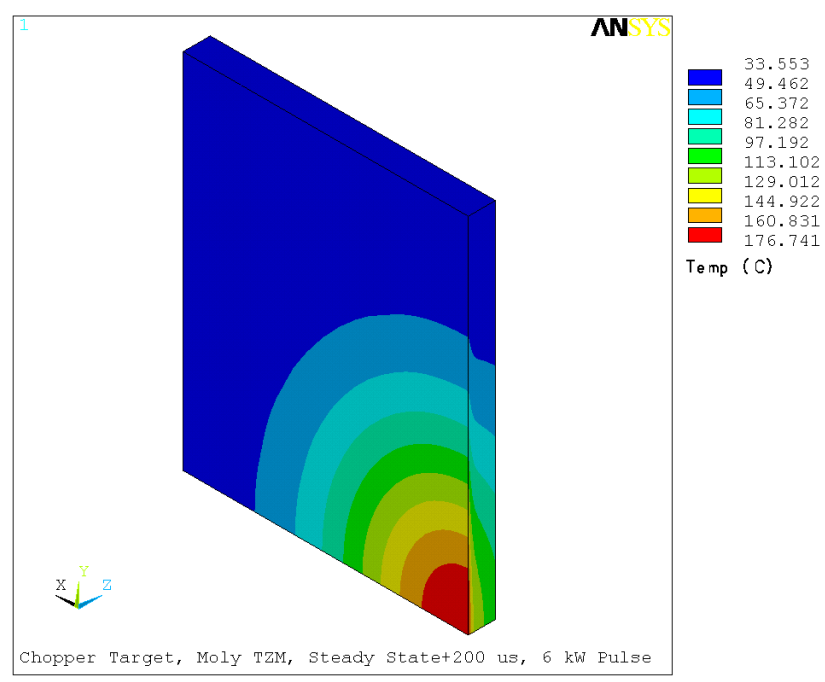

Figure 2: Typical temperature contour plot.

\subsection{Parametric Model Input}

In order to readily analyze a wide variety of devices and perform case studies, the primary input file which generates the model has been written to allow convenient modification of all key parameters. These include the beam power and spot size, the pulse length, the target material total thickness and cladding thickness, the angle of incidence and the element mesh densities. Other features of the model permit the user to specify solution from a cold start or a steady state condition, application of heat based on the stopping range profile or surface heating, and the use of temperature dependent or constant material properties.

\subsection{Mesh Density}

The computation of the model mesh densities is coded into the input file based on the range of the beam in the target, the size of the beam and the angle of incidence. At the target surface, the mesh is very fine in order to resolve the stopping range profile. The mesh then fans out to a larger size towards the rear surface. Similarly, the mesh density is high at the center of the bi-gaussian beam spot and decreases towards the outer edges of the model.

\subsection{Volumetric Heat Loading}

The calculation of the 3D volumetric heat load table is programmed within the model input file using the beam power and rms size in combination with the Bragg peak profile for the given material and beam energy. The Bragg peak defines the rate at which the beam energy is transferred as heat to the target atoms. This energy transfer increases as the beam penetrates the target surface, reaches a peak and then drops off to zero soon after. The stopping ranges for the materials of interest here are 20 to $30 \mu \mathrm{m}$ for the $2.5 \mathrm{MeV}$ beam. The Bragg peak profile is obtained by inputting the target material and the beam ion species and energy into the program SRIM (Stopping Range of Ions in Matter) [6]. The program was developed by IBM researchers to produce tables of stopping powers and range distributions for any ion at any energy. The resulting energy deposition data for the SNS Front End beam for numerous target materials of interest have been stored in a separate input file which is accessed by the primary model file.

\subsection{Target Cooling}

The finite element model described here simulates the actual cooling geometry by applying a uniform film coefficient over the entire rear surface. Most beam intercepting devices achieve heat removal by means of a series of narrow, deep cooling channels located near the surface. The channels function like cooling fins to remove heat more efficiently than by the method used in the model. To account for the difference, the model film coefficient must be increased to reach an equivalent level of cooling. The correct value is determined for a given material and geometry through the use of a $2 \mathrm{D}$ thermal submodel of a single cooling channel. The resulting heat transfer rate is used to calculate a corresponding coefficient for the model.

\subsection{Material Properties}

The temperature dependent thermal and mechanical property tables for all materials of interest are stored in a separate input file. A material number flag in the primary model input file identifies which set of properties is to be used. Since the material properties are not constant, the solution to the resulting non-linear FEA model is obtained iteratively within ANSYS.

\section{PARAMETRIC STUDIES}

A series of model runs was performed using different target materials, power levels and angles of incidence to determine their effect on temperature and stress. The intent of the analysis was to identify the material with the best beam intercepting properties and to determine to what degree a target can absorb additional power by means of shallow angles of incidence.

Figure 3 summarizes the results of a target material study for a $6 \mathrm{~kW}, 1 \mathrm{~ms}$ long pulse impacting a target at a $4: 1$ angle of incidence ( $\sim 76^{\circ}$ from normal). The beam size was $\sigma_{\mathrm{x}}=3.62 \mathrm{~mm}$ and $\sigma_{\mathrm{y}}=1.62 \mathrm{~mm}$. This case represents the MEBT chopper target which is used to sharpen the leading and trailing edges of the beam pulses. 
The comparison to yield is based on the strength of a given material at the peak target temperature as predicted by the model. The superior target material was found to be the molybdenum alloy TZM (Mo-0.5Ti-0.08Zr). Based on the results of this study, TZM is being used in all of the SNS Front-End beam intercepting devices.

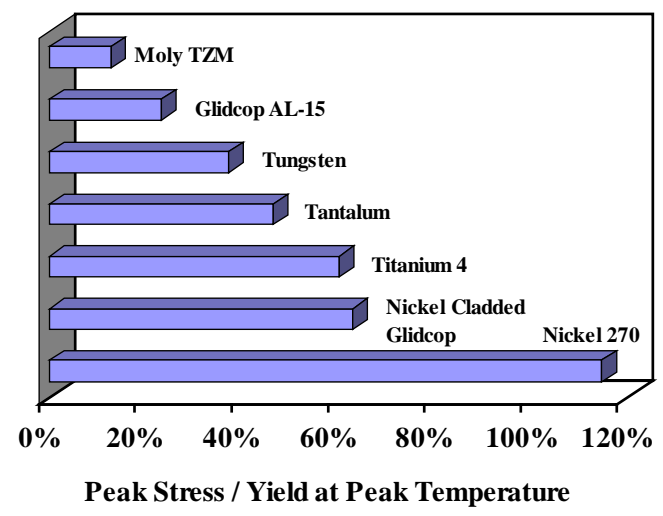

Figure 3: Target material stress comparison.

Another series of runs was performed to determine the peak stress in a TZM target as a function of both angle of incidence and pulse length. The curves plotted in Figure 4 represent the stresses on an emittance scanner slit heated by a $100 \mathrm{~kW}$ pulse with a spot size of $\sigma_{\mathrm{x}}=3.27 \mathrm{~mm}$ and $\sigma_{\mathrm{y}}$ $=4.16 \mathrm{~mm}$. As seen in the figure, the stress is dramatically reduced for shallow angles of incidence, thus allowing the target to absorb much longer pulse lengths.

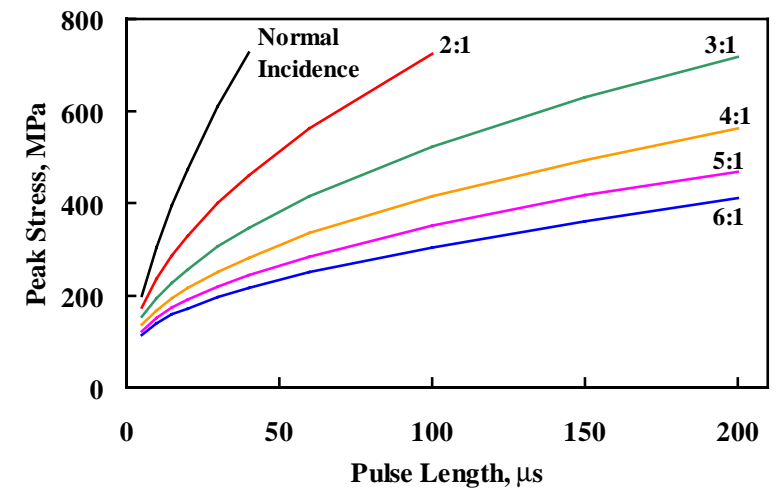

Figure 4: Peak stress vs. pulse length and angle.

\section{ENERGY DEPOSITION}

Many beam target models are simplified by applying all of the heat directly on the surface. However, in some cases, this method will cause the calculated peak temperatures and stresses to be significantly higher than the actual ones. A more accurate result is obtained by applying the heat according to the Bragg peak profile. A series of runs was performed to determine the degree to which the peak temperature rise is over predicted for surface heating at various pulse lengths and angles of incidence (Figure 5). The error is highest for shorter pulses since the Bragg peak method initially heats a larger volume than the surface heat method. For longer pulses, the diffusion of heat into the target dominates, and the initial distribution of the applied heat becomes less important. For shallow angles of incidence, the heat is deposited closer to the surface, resulting in less difference between the two methods.

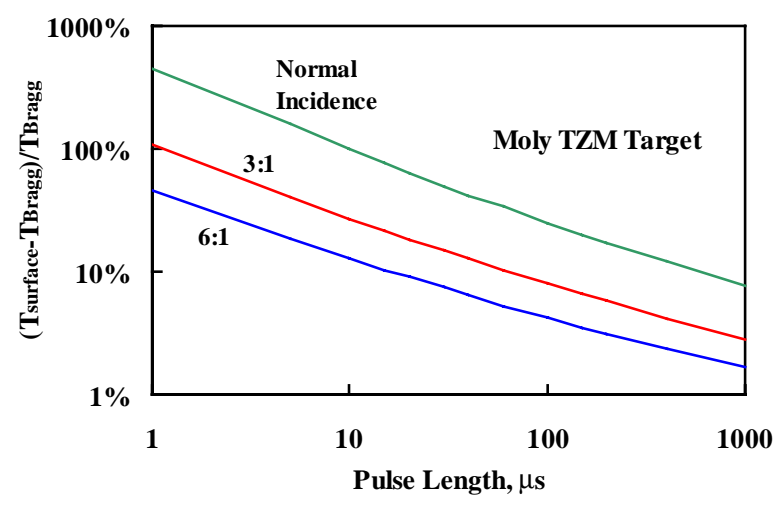

Figure 5: Bragg peak vs. surface heating.

\section{CONCLUDING REMARKS}

A parametric finite element model has been developed to analyze the induced temperatures and stresses in targets used to intercept high intensity beams. The 3-dimensional volumetric heat load applied to the model is based on a bigaussian beam shape which is deposited into the surface of the target according to a Bragg peak distribution. Through the use of the model, the SNS Front End Team has designed a beam chopper target, an emittance scanner slit and a beam stop. The beam stop operates under the most severe conditions, absorbing a $95 \mathrm{~kW}, 1 \mathrm{~ms}$ long pulse every $16.7 \mathrm{~ms}$. Fabrication of the devices is currently under way. The first tests with beam are expected to occur later in 2001.

\section{REFERENCES}

[1] R. Keller, "Progress with the SNS Front-End Systems", PAC '01, Chicago, June 2001.

[2] R. Thomae, P. Bach, R. Gough, J. Greer, R. Keller and K.N. Leung, "Measurements on the H-ion Source and Low Energy Beam Transport Section for the SNS Front-End System', Linac '00, Monterey, August 2000, 223-225.

[3] A. Ratti, R. DiGennaro, R.A. Gough, M. Hoff, R. Keller, K. Kennedy, R. MacGill, J. Staples, S. Virostek, and R. Yourd, "The Design of a High Current, High Duty Factor RFQ for the SNS", EPAC '00, Vienna, June 2000.

[4] A. Ratti, R.A. Gough, M. Hoff, R. Keller, K. Kennedy, R. MacGill, J. Staples, S. Virostek, and R. Yourd, "Fabrication and Testing of the First Module of the SNS RFQ', Linac '00, Monterey, August 2000.

[5] D. Oshatz, A. DeMello, L. Doolittle, P. Luft, J. Staples, and A. Zachoszcz, "Mechanical Design of the SNS MEBT', PAC '01, Chicago, June 2001.

[6] J. Ziegler, J. Biersack, and U. Littmark, "The Stopping and Range of Ions in Solids", Pergamon Press, New York, 1985. 